\title{
World Family Portrait A celebration of humanity's place in the world
}

\author{
RISC Consortium
}

In 2014, the RISC Consortium launched an ongoing interactive initiative entitled "A World Family Portrait." This call for contributions invites scholars, practitioners, journalists, photographers, etc. to submit written and photographic contributions in English, French, or Spanish that provoke a contemporary reflection on the human condition through the presentation and analysis of life challenges and opportunities. The goal of these publications is not simply to document world events/social conditions but to engage readers through photography and prose in a dialogue focusing on the evolution of our world and humanity's place in it. Selected photos will be published periodically in the Leadership Forum section of Regions $\mathcal{E}$ Cohesion. Interested contributors should go to the "World Family Portrait" link at www.risc.lu where texts and photographs can be uploaded along with basic information, such as author name(s), correspondence address, institutional affiliation, and current position. Articles should be approximately 500 words in length. Images should be submitted in a JPEG format at a resolution of approximately $1900 \times 1600$ pixels. All materials must be original. 


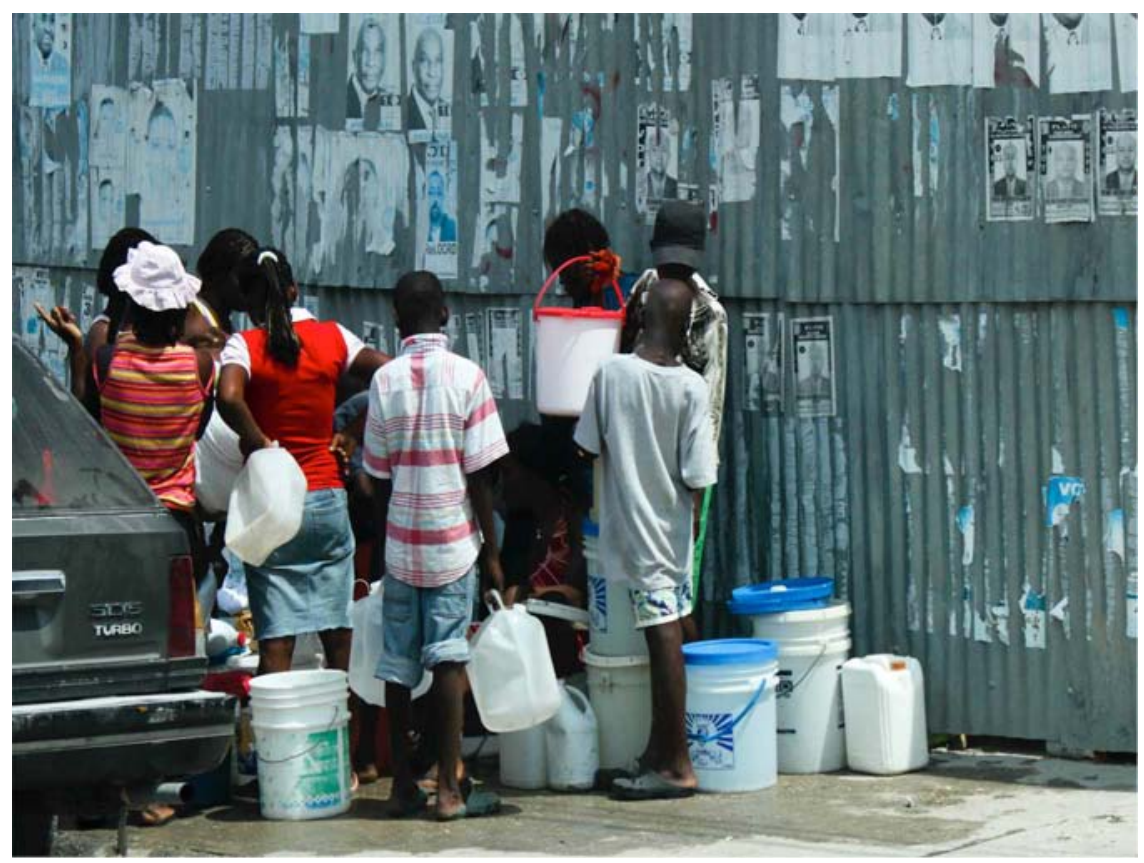

Figure 1 - Fetching water-Port au Prince, Haiti by Doug Metz

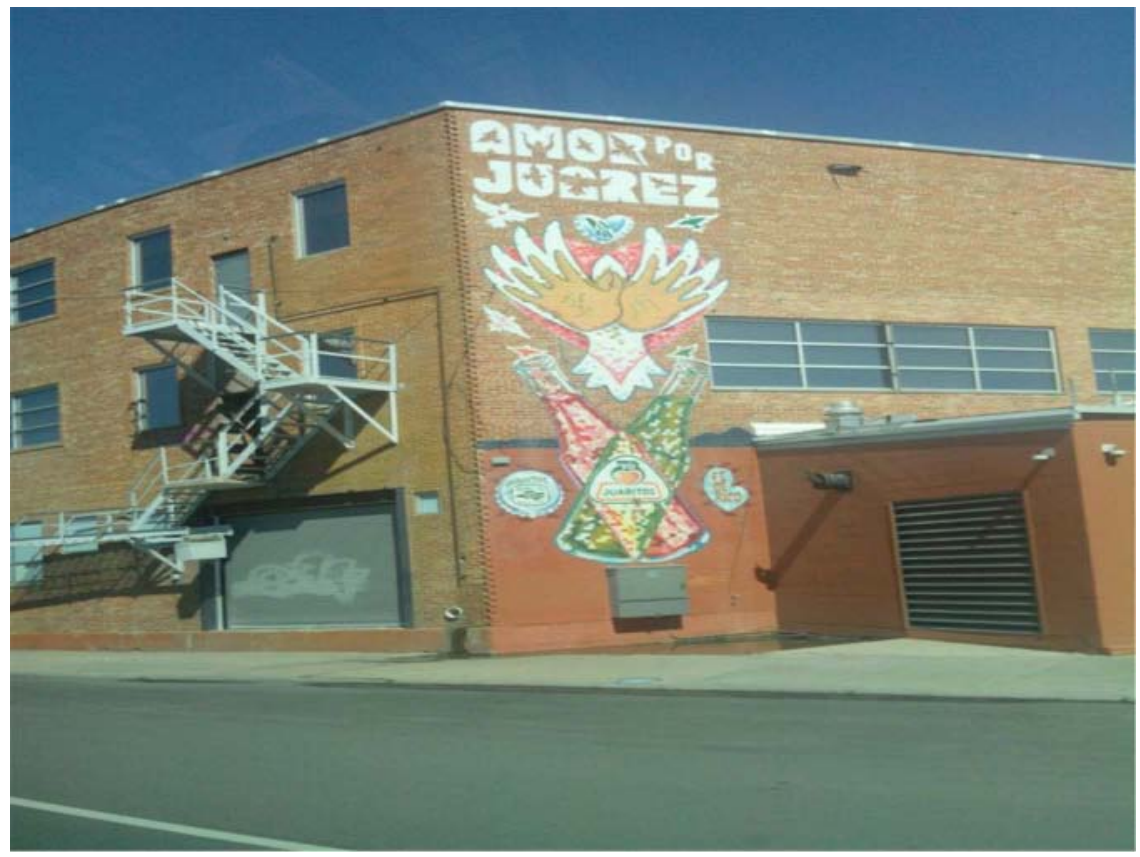

Figure 2 - Cross-border solidarity from El Paso, Texas, USA by Harlan Koff 


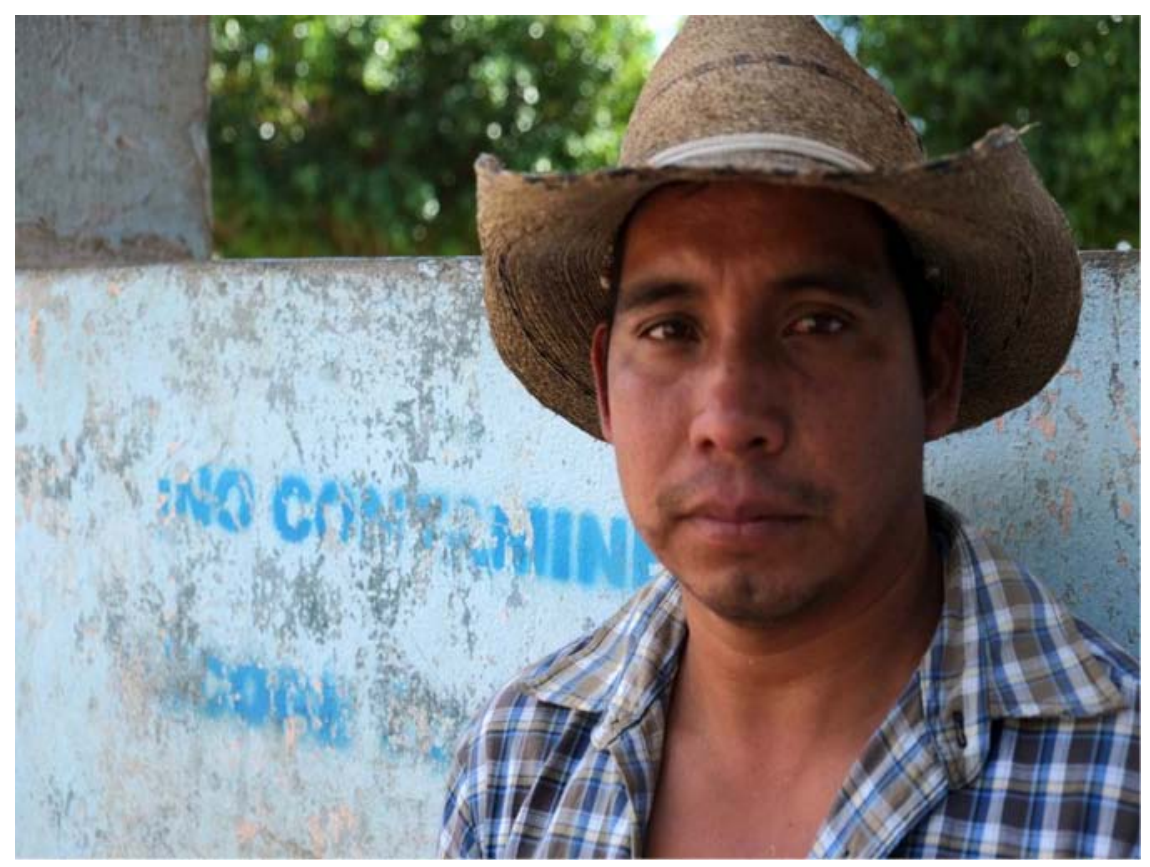

Figure 3 - Parole de l'eau, parole humaine by Carine Chavarochette

Janvier 2014, Mexique, Chiapas. Zone d'innombrables vestiges archéologiques mayas, de forêts plus tout à fait vierges, d'avancée de fronts pionniers, d'agrobusiness et d'extraction minière, de trafics en tout genre. Région frontalière stratégique, réservoir d'eau douce, l'État chiapanèque ne peut cependant proposer à tous ses habitants un accès à l'eau potable. Bien commun de l'humanité pour certains, marchandise pour d'autres, comment les populations perçoivent-elles cet élément minéral? À travers trois séries, 15 photos témoignent de la vétusté des réseaux d'adduction, du désengagement de l'État, de la pollution des sources et des rivières par les activités humaines, qu'elles soient domestiques, agricoles, industrielles ou minières. Pour les populations mayas, cet élément minéral reflète leur cosmogonie, pour toutes, il est à la fois, lien social et source de conflit. Par la parole de l'eau, ces images racontent des histoires singulières. À la suite d'entretiens réalisés, chaque personne interrogée a été photographiée. À la question: quel est votre rapport à l'eau (potable, fleuve, puits, source, réservoir, robinet, pluie)? Hommes et femmes ont sélectionné un lieu ou un objet qui symbolisait leur relation avec cet élément minéral. Par leur « vécu de l'eau » ces habitants interrogent le devenir de cette ressource. 1re série «Les déplacés du barrage de La Angostura » Lors de la construction du barrage hydroélectrique de La Angostura (entre 1969 et 1974), le vil- 
lage de dona F. et ses habitants ont été déplacés. Sa maison (celle que nous voyons sur les photos) a été démontée tuile par tuile puis reconstruite quelques kilomètres plus loin sur les terres collectives de l'ejido Laja Tendida. Les anciennes terres agricoles fertiles ont été inondées et les corps des ancêtres abandonnés au fond du lac artificiel. Cette femme demeure attachée à sa citerne, sa pila, qui lui sert encore de réservoir lors des coupures d'eau en saison sèche. 2e série "L'eau (saine) est un droit » Deux générations de leader communautaire sont représentées ici. La première s'est battue pour que l'État lui donne des terres et les moyens d'assurer la survie de sa famille. Parallèlement, cette génération a négocié avec les divinités telluriques pour l'obtention de pluies sur leurs semences. La seconde tente de conscientiser les habitants sur les risques de contamination de l'eau et voudrait que les pouvoirs publics légifèrent sur le rejet des eaux usées et des déchets toxiques dans les rivières afin de freiner la recrudescence des maladies infectieuses. 3e série « Réseau de distribution de l'eau, un enjeu municipal et électoral à Benemerito de las Americas » Responsable du bureau municipal des eaux de la ville frontière de Benemerito de las Americas, politique plus qu'ingénieur, cet homme doit assurer un réseau de distribution d'eau potable à l'ensemble de ses administrés. Il doit veiller à l'entretien des canalisations existantes, vétustes et incomplètes, développer un service de traitement des eaux usées et éviter autant que possible la pollution de deux fleuves (Lacantun et Usumacinta) entourant sa ville, par les déchets toxiques ou les eaux domestiques. 


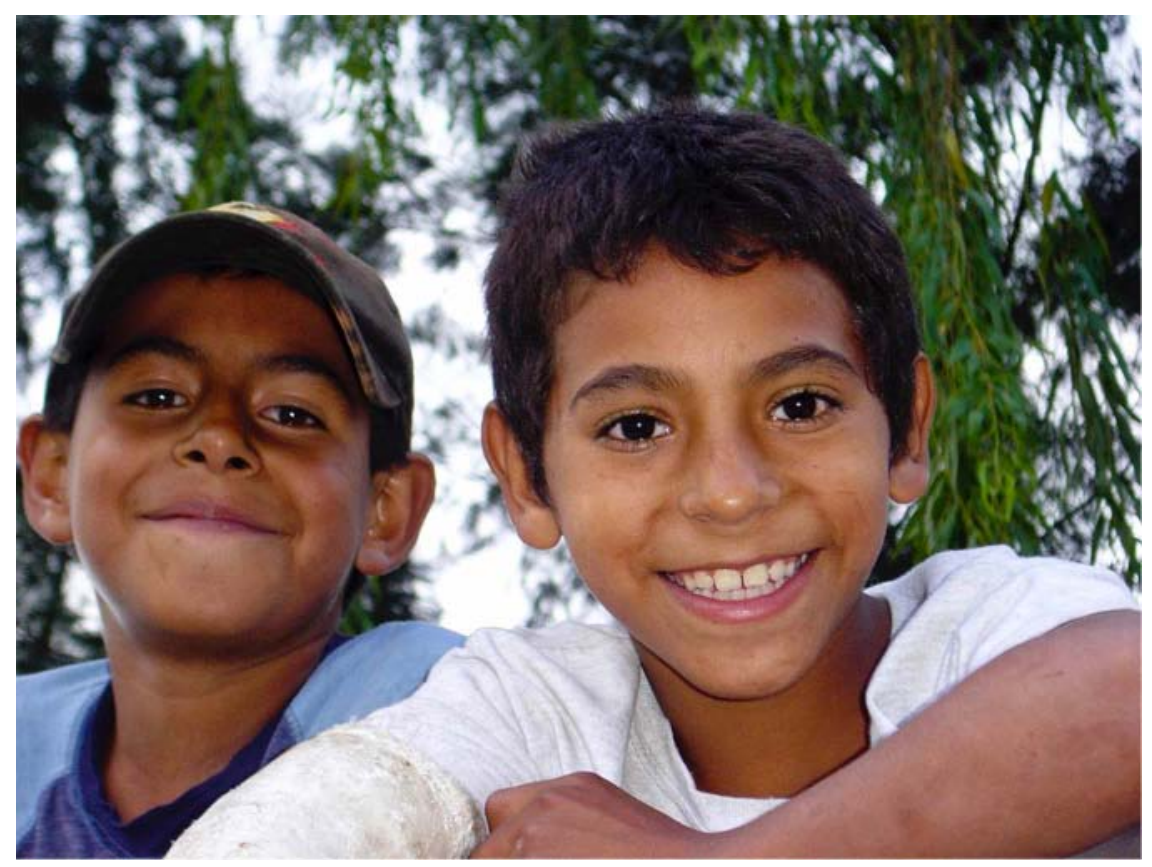

Figure 4 - Friends for life-Honduran school kids by Doug Metz

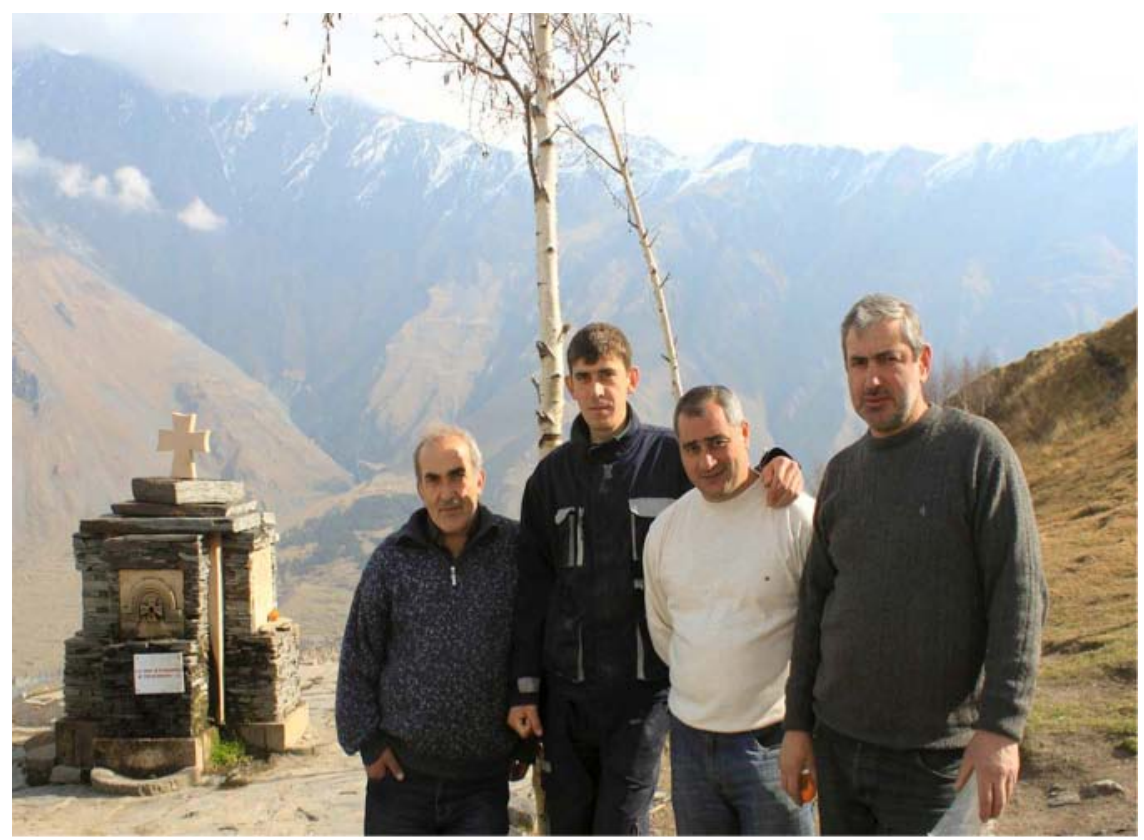

Figure 5 - Celebrating holy day at mountaintop Gergeti Sameba Church, outside Kazbegi, Georgia by Doug Metz 